\title{
The Dinosaur in the Classroom: what we stand to lose through ability grouping in the primary school
}

\author{
RACHEL MARKS
}

ABSTRACT Embedding setting (subject-based ability-grouping) into the primary-school environment creates structural conflict - physically and culturally - fundamentally changing the nature of primary schools through the imposition of secondary practices and cultures and the loss of pastoral care. This article examines the hidden implications for teachers and pupils of taking on secondary-school roles within the primary-school context. It highlights the wide-ranging, yet nuanced impacts of the use of setting, examining the shift towards subject-based thinking and the erosion of the pastoral-centred holistic ethos of primary education.

\section{Introduction and Context}

In an earlier article (Marks, 2013) I explored how fixed-ability thinking persists in the absence of structured ability-grouping practices. ${ }^{1}$ In this article I return to the concept of hidden consequences, exploring how setting may inadvertently be a contributory factor in the loss of traditional primary school relationships.

Recent research suggests that the use of ability-grouping is increasing in England's primary schools (Hallam and Parsons, 2013) despite limited research into its effects. Ten years ago, Davies et al $(2003$, p. 46) reported that 'there has been little recent primary school-based research in the UK into the effects of grouping by ability'; this situation has not changed substantially. Research tends to address three areas: group allocation, attainment and attitude. From the 1960 s to the present day, concerns have been raised that ability-group allocation has been influenced by teacher-judgement. Factors such as; social class, free-school meals status, English language learners, special educational needs and month of birth have been linked with ability-group placement. Studies into the impact of ability-grouping on attainment and attitude paint a fairly consistent picture; one of no overall impact on attainment and variable, inconsistent, impacts on attitudes. Beyond attainment and attitudinal outcomes there has been limited work into ability-grouping in England's primary schools, yet it is important to consider the specifics of the primary school, an environment less restricted by subject or age boundaries but with a particular approach and ethos.

This article considers ability-grouping beyond the oft examined areas of attainment and attitude. Taking data from a longitudinal study into ability-grouping and Discourse in the primary school (Marks, 2012), it debates some implications of ability-grouping that may go unnoticed by practitioners and which do not have a place in traditional research reports. As such, the article is not a research report in the traditional sense but draws on a set of inter-linked themes arising from a wider study which do not have an easy place within other writing but which are nonetheless important to discuss. 


\section{The Research Study and Schools}

The wider research involved a longitudinal study of ability practices and discourses in two primary schools - Avenue Primary and Parkview Primary ${ }^{2}$ - in greater London. The research focused principally on mathematics classes as a field in which ability-grouping predominantly occurs. The study involved 284 pupils and their teachers in Year 4 (ages 8-9) and Year 6 (ages 10-11, the final year of primary schooling). Further details of this mixed-methods study can be found in Marks (2012, 2013, In Press).

This article draws on data from pupils experiencing setting at both Avenue Primary and Parkview Primary. Parkview Primary was described in the earlier article (Marks, 2013). With nearly 450 pupils and 19 teachers, Parkview Primary serves a diverse community with pupils coming from both local housing estates and large detached properties further from the school. A third of pupils (compared with $21 \%$ nationally) are eligible for free school meals. Whilst the use of ability-grouping practices was rather more complex than would first appear, setting was only used in Year 6 and only for mathematics. Avenue Primary has more explicit ability-grouping practices. This school is a highachieving, over-subscribed school ( $90 \%$ of pupils achieved or exceeded the expected Level in mathematics in the year of the study compared with $79 \%$ nationally) located in an area of high socioeconomic status with $10 \%$ of pupils eligible for free school meals. The school has almost 700 pupils, 35 teachers and 40 teaching assistants. At Avenue Primary, pupils are rigidly grouped into one of four sets for mathematics from Year 2 (ages 6-7).

The wider study drew on constructivist grounded theory (Charmaz, 2006) leading to the development of four over-arching themes: constructing, enacting, experiencing and labelling ability. Between and across these themes, sub-themes also arose. This article draws on these sub-themes, presenting some of the hidden implications of setting. The following sections are structured around three of these sub-themes, exploring how aspects of setting are experienced by teachers and pupils across both schools.

\section{At the Beginning of the Day}

The traditional image of the primary school in England is one organised on a classroom / classteacher based system with one generalist teacher per class, teaching the pupils for the majority of subjects (perhaps with the exception of specialist input for subjects such as music and Modern Foreign Languages) across the school day. This structure sees teachers involved with their pupils at many non-subject based times from routine administration in taking the register, through accompanying their class to assemblies, maintaining vigilance on playground duty, to possibly eating lunch with their class and organising after-school activities. England is particularly unique in terms of its approach to pastoral care, with the child's welfare central to the role of the primary school teacher (Broadfoot et al, 1987). Unlike primary education systems in some other countries where the teachers' focus is subject-based and academic (see, for example, the systems in France and Russia, Alexander, 2000; Osborn, 2001) and where pastoral issues are not generally part of the cultural tradition, primary school teachers' work in England involves a significant pastoral component. 
The relationships developed through this position of care are important considerations in research examining practices - such as ability-grouping - which fracture this traditional role. As has been reported elsewhere (Marks, In Press), setting exposes a rupture between the physical set up of the primary school, designed for each class to stay within its base for the majority of the day, and the pupil movements arising from relocation to group teaching rooms. The implications of pupils' movement away from their class teacher for setted lessons were evident both at Avenue Primary and Parkview Primary.

At both schools, particularly where pupils were set for the first lesson of the day, the beginning of the day did not resemble the traditional beginning of a primary school day but a rushed and stressful time as teachers attempted to settle the class and deal with daily administration tasks before pupils had to move to other classrooms. The use of setting imposes a rigid timetable on teachers and classes where the set lesson must start and finish at a specified time. In the absence of setting primary teachers can make decisions to rearrange their timetable in response to emerging needs. If the class are unsettled due to an external incident the class teacher can make time to address this. If a pupil arrives at school in a distressed state, this can be acted on immediately. However, where setting imposes an urgency to send pupils to other teachers and a new group of pupils are arriving outside the classroom-door, the space for pastoral care is restricted. As was observed in this study, pupils are sent to set lessons in distressed states without communication of this to the set teacher and pupils at times find it difficult to engage within sets as they are struggling to make sense of other events; events which may seem trivial, such as falling out with friends, but which impact on pupils' engagement.

\section{Displacement and Disruption}

Through removal from a class-based learning environment, setting, which, even in the absence of bells, must happen at rigidly controlled times, displaces pupils and causes disruption to established teacher-pupil and pupil-pupil relationships. Pupils are moved away from their peers and from the class-teacher they have developed a relationship with. It is important to remember that pupils' class and external relationships still remain even if momentarily broken for the duration of a set lesson, yet setting often fails to account for the relationships pupils bring, with these apparently ignored when a pupil enters a set classroom. The strength and impact of these forgotten relationships was exemplified when pupils were asked about how they felt during set mathematics lessons. Much of what pupils talked about related to incidences and relationships located outside of, but which impacted on what happened within, the mathematics classroom:

Rhiannon: If it was after play and I had fallen out with my friend and then I had to go to maths I would feel a bit upset, because other things happen before the maths, it's not just the maths that makes me happy or sad.

Wynne: Maths before lunch I'm not very good with because I am having bad lunchtimes and I have to go and tell Mr Iverson when I am feeling fine and when I am not. I normally like maths before break or after lunch, but not before lunch.

Both Rhiannon (Year 6) and Wynne (Year 4) at Avenue Primary talked about the impact of broader school events on their learning in mathematics. Wynne's class-teacher, Mr Iverson, was aware of the 
difficulties she was facing in maintaining positive peer relationships, yet was unable to give consistent pastoral support because Wynne was extracted from his care for mathematics and they were not in contact at key moments of the day such as before lunchtimes.

In addition to school-based events, a primary teacher's pastoral role includes being aware of how pupils' wider lives impact on their learning. However, there may be limited time for class-teachers to share this information with set-teachers, or limited capacity for set-teachers to hold and respond to such information. At Avenue Primary, one bottom set Year 4 pupil, Yolanda, was often late for mathematics and appeared disengaged. Yolanda's set teacher attributed her disengagement to a lack of ability and Yolanda was often reprimanded for not paying attention. However, speaking to Yolanda's class-teacher revealed a chaotic home-life, something Yolanda touched on when I interviewed her and asked her to draw and talk about what she thought about in mathematics lessons:

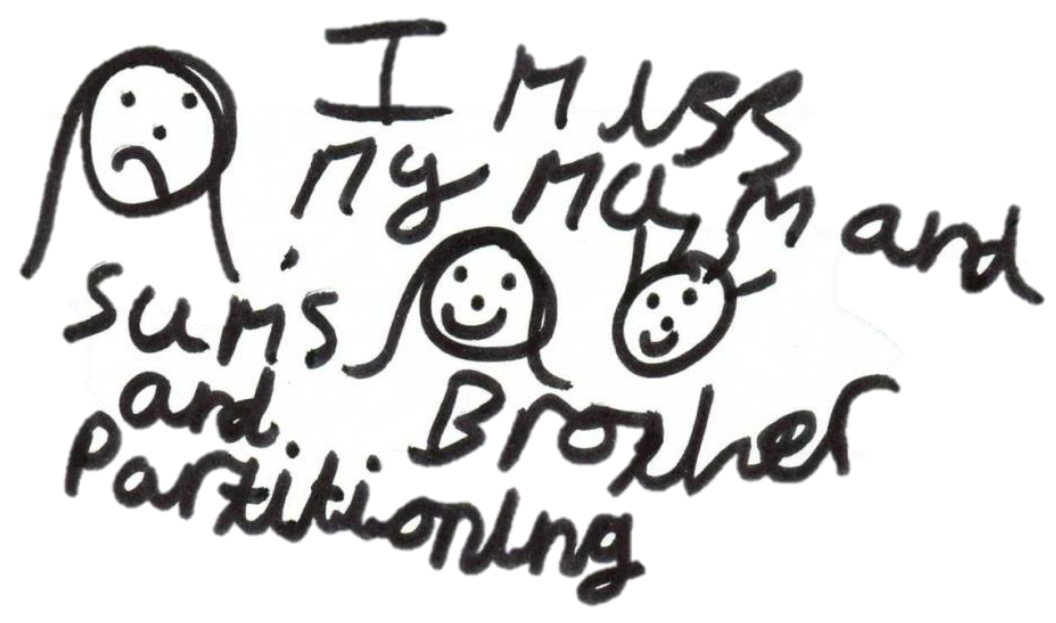

Discussing her picture, it was clear that whilst some mathematics was part of her thoughts (sums and partitioning) Yolanda was preoccupied with external events:

Yolanda: Sometimes I miss my mum and my brother, it's just sometimes that pops into my head in maths because my brother is quite cute and my mum always gives me cuddles. I miss that. Sometimes I think about sums in maths.

The limited opportunity for the set-teacher to engage with pupils beyond the mathematics teaching meant that the significant role of Yolanda's home-life was ostensibly, although unintentionally, ignored. The breakdown of pastoral support, as a hidden consequence of setting, may have contributed towards more negative impressions being formed of pupils and may have restricted learning opportunities as set-teachers, working under a fixed-ability mindset, see a lack of ability rather than pupils facing external barriers to learning.

\section{Subject Teachers or Primary Teachers?}

When a teacher teaches a set rather than their own class a shift occurs from a holistic to a subjectbased focus. Pupils move to a set for a subject (e.g. mathematics) with a subject teacher who they may only encounter in that context. Whilst class-teachers develop a knowledge of their pupils that encompasses the whole school day across subjects and extra-curricular activities, set teachers only 
know pupils in a subject-specific context. Although the set teacher's understanding of the pupil in that subject may be strong, they are limited in understanding pupils across the curriculum.

Within this study teachers were asked to reflect on the differences between teaching mathematics as a class-subject and within setting arrangements.

Mr Donaldson: Well it's, I don't know, it's got more, they're your children, they're your class and you know where they are across the curriculum and you know, I think that in itself can help me to understand, you know where to push them on a little bit more, but if you see them for an hour every day it's just more difficult.

Mr Donaldson, introduced in the previous article, taught a Year 4 mixed-ability class for all subjects at Parkview Primary. Previously he taught in schools using setting for mathematics and so was able to contrast his experiences of teaching within both structures. In the extract above, Mr Donaldson acknowledged the difficulties associated with setting whereby he felt there were restricted opportunities to engage fully with each child across the curriculum when only teaching pupils for a rigidly demarcated portion of the day.

Mr Donaldson also talks about being better positioned to support pupils' learning when he sees them across the curriculum. This reflects a traditional primary school approach which responds flexibly to pupils' needs, drawing on children's ideas and imaginations. Often primary teachers will thread a theme across curricular areas helping pupils to make connections and utilising their interests to sustain engagement. Where setting splits the day up into subjects, these opportunities are diminished. This was illustrated when interviewing Zackary, a Year 4 pupil in the bottom set at Avenue Primary. The interview started along the normal lines. I asked Zackary what he thought about in mathematics lessons and he provided a fairly standard answer rooted in school mathematics:

Zackary: What is the answer and how to get there.

However, this is where the school mathematics ended, as Zackary continued unprompted:

Zackary: And then there's this. I think about this all the time.

At this point, Zackary became engrossed in drawing me an image of a robotic dinosaur, 'Pleo', enthusiastically regaling me with details of its development:

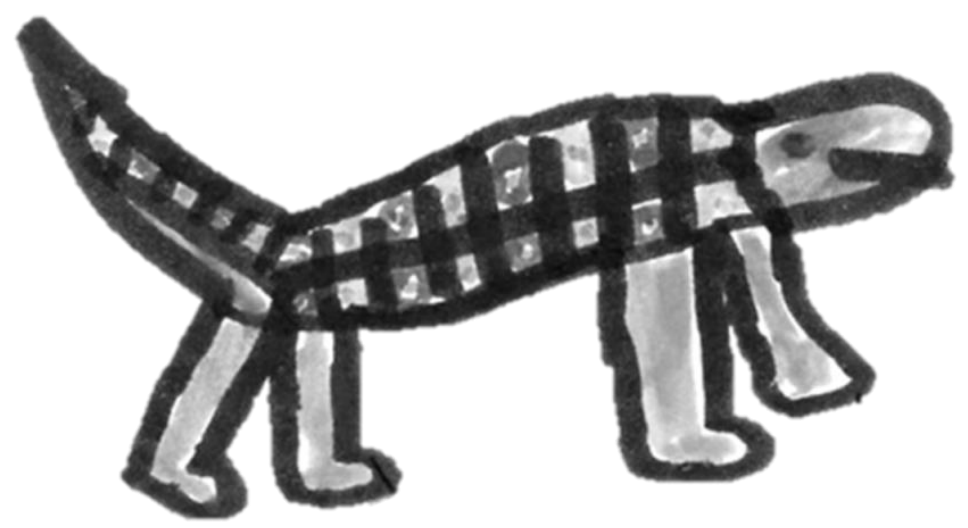


Zackary: It's not my best drawing; it's got a bit mixed up. It's a robot and it's in the Argos catalogue and it's called Pleo and it costs $f 250$ but I'm still going to get it and I really really want it and it's going to be my biggest Christmas present ever and I'm only going to get that for Christmas and that's it. I'm thinking about it all the time, when I'm going to bed, when I get up in the morning. I think more about that than the maths. It's the most lifelike robot that anyone has ever made in the world. It makes noises, it interacts with you and it is exactly the same size as a baby komodo dragon. An adult komodo is about from the ceiling to down there and from about there to there but a baby would be about that and that's the size it would be. It took 4 years to make because they had to make the head the right size, but if they make it bigger that would increase the power but then in the motor there they would have to put more power in there and that would affect the leg power and that would affect the tail power. They had to work for four whole years, that's a very long time. I don't know if there will be a delay because it was supposed to come out in September but there was something wrong with its charger because it needs to be charged for 8 hours.

In this extract Zackary is engaging with some quite complex mathematical ideas involving ratio and proportion, time, and measurement, using ideas and quantities in excess of the one and two digit, manipulative-supported, calculations he was involved in in his set mathematics lessons. However, in thinking about this dinosaur rather than engaging with the school mathematics, Zackary was constructed by his set mathematics teacher as disengaged and of low mathematical ability. Had Zackary been taught by his class-teacher there may have been the opportunity to elicit his interest and use it across curriculum areas - using the robotic dinosaur as a context for writing, mathematics, or design work - with the potential to engage Zackary and present a very different construction of him as a learner. There is of course no guarantee that this would happen in the absence of setting as teachers work to balance the interests of pupils across their class, but setting represents a very real barrier to utilising a cross-curricular approach.

The subject-based approach unavoidable in setting also changes how teachers view learners. A subject focus means that pupils have limited ways in which to be viewed as successful. Teachers are reduced to thinking in terms of the subject and view success in these limited terms. As observed in this study, pupils were expected to switch off the outside world with the most successful mathematics pupils considered to be those who could compartmentalise, or at least project an image of having compartmentalised, their identities, displaying only their mathematics learner identity. This is illustrated below by Uma and Victoria, both in the top-set in Year 4 at Avenue Primary. In this interview extract we were talking about what makes someone good at mathematics and I had asked them to list and discuss things that might lead to a child doing well:

Uma: $\quad$ And thinking, I think that comes here. If you're thinking you might work harder.

Victoria: $\quad$ But you might be thinking about something else.

Uma: Well thinking about the lessons. Thinking about things that aren't the lesson won't help. We're in the top-group because we're not always thinking about other stuff.

Victoria: If you're not thinking about the lesson then you're going to get it wrong. Because most people who talk a lot, that's like me, but then when it sort of 
comes to the lessons I pay attention. Paying attention, we can put that on as well.

Uma: $\quad$ Also I think you should try and, you know, if you have an argument at playtime, you should try and forget about that while you are lining up because otherwise your mind will be buzzing round with 'ooh, maybe they're not my friend any more, will they be my friend at lunchtime because of what happened today', you have to try and forget that and instead focus on the maths, focus on the lesson.

Victoria: Yeah, you have to try and forget about anything that is worrying you. Worrying things.

Contrasting with Rhiannon and Wynne earlier, Uma and Victoria identify that success is viewed in terms of being able to switch-off the world outside of the mathematics classroom. The subject focus of setted lessons allows this to happen in a way that may not be so obvious in the more traditional primary classroom. Uma and Victoria both appear to have learnt to project a particular identity - a bland version of their wider selves - in order to be considered successful within set lessons.

\section{Discussion}

This article highlights some hidden implications of setting in the primary-school environment. It represents an important addendum to the body of studies into ability-grouping. This article suggests how ability-grouping may have multiple impacts on pupils, well beyond those expected or easily observed. The ability-grouping literature notes that the iniquitous outcomes of setting are driven by differential teaching experiences from repetitive, conceptually simplified, work in low-sets to fastpaced competitive environments in top-sets. This article suggests how ability-grouping - in this case setting - may have more nuanced impacts, fundamentally changing the nature of primary-schools. This work sits together with the previous article examining mixed-ability classrooms to suggest how an orthodoxy of fixed-ability has impacts beyond those directly attributable to ability-based practices.

Many issues discussed in this article arise due to structural mismatch. Setting attempts to introduce a structure from secondary education, grounded in individual subjects, into the primary environment, grounded in holistic learning. Primary schools are not physically suited, nor are primary pupils socially mature enough, to cope with setting and the inevitable subject cultures, particularly where this significantly reduces pastoral input.

Setting requires primary pupils to assume the roles and responsibilities of secondary-school pupils. They move around the school for different subjects, respond to different teachers and work with different peer groups. These are quite extensive - and potentially confusing - demands to make of primary-aged pupils. Being placed in a secondary education set-up pupils are exposed to iniquitous practices with incumbent socialisation into beliefs about, and acceptance of, innate ability differences, from an early age.

It is important to emphasise that the teachers in this study were not deliberately acting in ways contrary to effective primary school teaching, but were responding to the consequences of a common classroom practice. Where they were aware of the impacts of these practices - such as 
imperfect pastoral care - they sought to ameliorate these, providing alternative, albeit more deprived, opportunities for enacting pastoral care. However, it cannot be denied that setting changes the traditional teaching approaches of the primary school. These practices are not going to be eradicated overnight. As such, it is important that ways are developed to work with these practices, identifying and responding to their widest outcomes.

There needs to be clarity about the purpose of primary education. Should the traditional pastoral role be retained or is there an argument for a more secondary-like subject-demarcated approach? Presently, schools face a mismatch and it is the uncertainties and unexpected consequences of this that are problematic. Teachers in this study talked about wanting to fulfil a traditional role, providing pastoral support and considering pupils' development holistically, but simultaneously they could not escape what they felt were extensive external pressures to perform a subject role with the belief that this maximized attainment. The teachers in this study talked about valuing the time they were given away from the classroom to talk in their interviews. Time for reflection needs to be a feature of the tightly timetabled lives of teachers in order to open-up discussion about the purpose of primary education. Establishing such dialogue may provide a way in to addressing the implications highlighted in this article.

\section{References}

Alexander, R. J. (2000) Culture and pedagogy: International comparisons in primary education. Oxford: Blackwell.

Broadfoot, P., Osborn, M., Gilly, M., \& Paillet, A. (1987) Teachers' conceptions of their professional responsibility: Some international comparisons. Comparative Education 23(3), 287-301. doi:10.1080/0305006870230304

Charmaz, K. (2006) Constructing grounded theory: A practical guide through qualitative analysis. London: Sage.

Davies, J., Hallam, S., \& Ireson, J. (2003) Ability groupings in the primary school: Issues arising from practice. Research Papers in Education 18(1), 45-60. Doi: 10.1080/0267152032000048578

Hallam, S., \& Parsons, S. (2013) Prevalence of streaming in UK primary schools: evidence from the Millennium Cohort Study. British Educational Research Journal 39(3), 514-544. http://dx.doi.org/10.1080/01411926.2012.659721

Marks, R. (In press) Educational Triage and Ability-Grouping in Primary Mathematics: A Case-Study of the Impacts on Low-Attaining Pupils. Research in Mathematics Education, 16(1).

Marks, R. (2013) 'The Blue Table Means You Don't Have a Clue': The persistence of fixed-ability thinking and practices in primary mathematics in English schools. FORUM, 55(1), 31-44. http://dx.doi.org/10.2304/forum.2013.55.1.31

Marks, R. (2012) Discourses of ability and primary school mathematics: Production, reproduction and transformation. Unpublished PhD Thesis. King's College London. 
Osborn, M. (2001) Constants and contexts in pupil experience of learning and schooling: Comparing learners in England, France and Denmark. Comparative Education 37(3), 267-278. doi:

$10.1080 / 03050060120067776$

\section{Acknowledgements}

I would like to thank the anonymous schools, pupils and teachers for their commitment to this study. This research was funded by a studentship from the Economic and Social Research Council (award number: PTA-031-2006- 00387).

RACHEL MARKS is a Senior Lecturer in mathematics education at the University of Brighton. She completed her ESRC funded doctorate entitled 'Discourses of Ability and Primary School Mathematics: production, reproduction and transformation' in 2012. Her research interests include primary mathematics, ability-grouping and equity issues. Correspondence: r.g.marks@brighton.ac.uk

\footnotetext{
${ }^{1}$ As in the previous article, the term ability is used without quotation marks to aid readability. However, the reader should assume that the legitimacy of the concept - and its associated assumptions - is continually in question and challenged throughout.

${ }^{2}$ All names are pseudonyms.
} 